\title{
Asociación de la Atorvastatina Sistémica en las Caracte- rísticas Clínicas Inflamatorias de la Periodontitis Crónica
}

\author{
Association of Atorvastatin in Systemic Inflammatory Characteristics of Chronic Periodontitis
}

\author{
J. Bravo-Bown* \& M. Ulloa-Carmona**
}

BRAVO-BOWN, J. \& ULLOA-CARMONA, M. Asociación de la atorvastatina sistémica en las características inflamatorias de la periodontitis crónica. Int. J. Odontostomat., 9(3):493-496, 2015.

RESUMEN: Determinar el efecto de la atorvastatina sistémica en el índice gingival y el índice de carga inflamatoria periodontal (PIBI), en pacientes con periodontitis crónica en una población adulta del sector norte de la ciudad de Antofagasta. Este estudio se realizó en pacientes derivados a la clínica de periodoncia del Centro Asistencial Norte Antofagasta, departamento dependiente del Servicio de Salud Antofagasta. Se seleccionó un grupo de 60 pacientes, mayores 40 años de edad, diagnosticados con periodontitis crónica, con al menos 14 dientes en la cavidad oral y sin tratamiento antibiótico en los últimos 6 meses. Se dividieron estos pacientes en dos grupos; 30 de ellos en tratamiento con atorvastatina por al menos 1 año (Grupo I) y 30 que no estaban en tratamiento con este medicamento (Grupo II). Las variables analizadas fueron el índice de placa bacteriana simplificado, el índice gingival y el PIBI, de la abreviación del inglés Periodontal inflammatory burden index. La atorvastatina sistémica se asocia a una disminución en el índice gingival y el PIBI, en pacientes adultos con periodontitis crónica.

PALABRAS CLAVE: inhibidores de la hydroxymethylglutaryl-CoA reductasa, índice periodontal, enfermedad periodontal.

\section{INTRODUCCIÓN}

Las estatinas son un grupo de medicamentos frecuentemente utilizados en el tratamiento de la dislipidemia. Pertenecen a la familia farmacológica de los inhibidores de la 3-hidroxi 3-metilglutaril-coenzima A reductasa. Su principal efecto y razón de su amplia utilización es que son capaces de reducir los niveles de colesterol séricos, principalmente las lipoproteínas de baja densidad (LDL) disminuyendo la incidencia de eventos ateromatosos (Ikeda \& Shimada, 1999). Además presentan un efecto antiinflamatorio, a través de la inhibición del antígeno 1 (LFA-1), receptor que al ser inactivado disminuye la función de los linfocitos T, macrófagos y polimorfos nucleares neutrófilos (Ikeda \& Shimada).

La periodontitis es una enfermedad infecciosa, inmunoinflamatoria y de carácter multifactorial que posee como factor etiológico la placa bacteriana, genera grados importantes y mantenidos de inflamación gingival y, destrucción de tejido conectivo y hueso alveolar, con la subsecuente formación de saco periodontal, pérdida de inserción clínica, llegando en algunos pacientes a generar pérdida dentaria (Kinane, 2001).

En estudios realizados en animales por Vaziri et al. (2007) y Nassar et al. (2009), se señala que las estatinas influencian el curso de las enfermedades periodontales disminuyendo las características clínicas inflamatorias y el grado de destrucción ósea. En un estudio hecho por Cunha-Cruz et al. (2006) realizado en humanos con periodontitis, y tratados con estatinas se encontró una menor incidencia de pérdida dentaria y estableció que el grado de severidad de la enfermedad periodontal, usando como variables, índice gingival, profundidad de sondaje y pérdida de inserción clínica se encontraban disminuidos.

\footnotetext{
* Especialista en Periodoncia, Profesor Asociado, Universidad de Antofagasta, Antofagasta, Chile.

** Especialista en Periodoncia, Profesor Asistente, Universidad de Antofagasta, Antofagasta, Chile.
} 
El presente estudio tiene por objetivo determinar el efecto de la atorvastatina (estatina sistémica) en pacientes con periodontitis crónica. Las variables estudiadas fueron el índice de placa bacteriana simplificado, índice gingival e índice de carga inflamatoria periodontal.

\section{MATERIAL Y MÉTODO}

Previa autorización de la dirección del Servicio Asistencial Norte se recolectaron las fichas clínicas de 60 pacientes, 30 de ellos en tratamiento con atorvastatina sistémica mayor a $10 \mathrm{mg}$ por un periodo superior a un año y 30 sin ingesta del medicamento. Fueron incluidos pacientes con: i) presencia de periodontitis crónica severa (Armitage, 1999), ii) mayor a 40 años, iii) presencia de más de 14 dientes, iv) índice de placa bacteriana superior al $50 \%$ (promedio en cavidad oral), v) sin ingesta de antibióticos en los últimos 3 meses previo al examen y vi) sin presencia de hábito tabáquico.

Las variables de interés estudiadas fueron: el índice de placa bacteriana simplificado (de O'Leary), el índice gingival (IG) (de Löe \& Silness) y el índice carga inflamatoria periodontal (PIBI) (Lindy et al., 2008b). Los materiales utilizados fueron los siguientes, pastilla reveladora de placa bacteriana (marca Sanoral 2tone), sonda periodontal OMS (Hu-Friedy), espejo oral ( $\mathrm{N}^{\circ} 5$ Aone).

Los pacientes fueron evaluados clínicamente por un solo examinador que desconocía el grupo al cual pertenecía cada paciente (ciego simple).

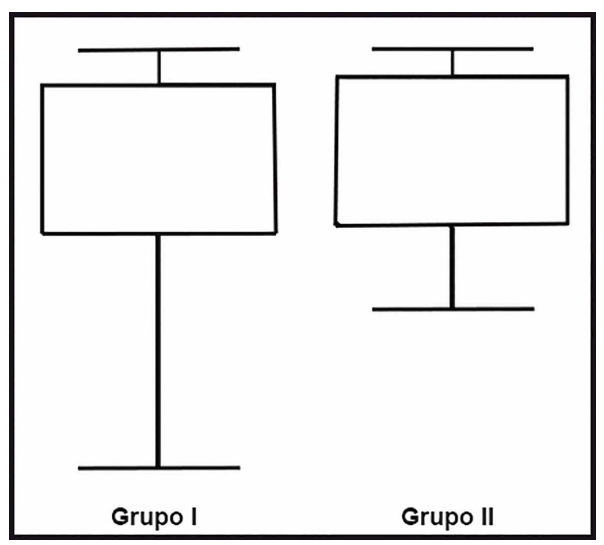

Fig. 1. Comparación en el Grupo I (izquierda) y Grupo II (derecha) según índice de placa bacteriana (promedio cavidad oral).
Los datos no presentaron distribución normal (test Anderson-Darling) ni varianzas homogéneas (prueba de Fisher) por lo que se usaron pruebas $U$ de Mann-Whitney para evaluar posibles diferencias de los índices (IG, PIBI) entre grupos. Los análisis se realizaron con el programa Statistica 7.0.

\section{RESULTADOS}

El rango de edad y la cantidad de placa bacteriana (Fig. 1) de los pacientes incluidos en el estudio no presentó diferencias significativas (Tabla I).

El grupo en tratamiento con atorvastatina (Grupo I) presentó menor cantidad de inflamación gingival, medido según el índice gingival $(1,75 \mathrm{v} / \mathrm{s} 1,91)$ y menor valor de destrucción periodontal según índice de carga inflamatoria (PIBI) (Tabla I, Figs 2 y 3 ).

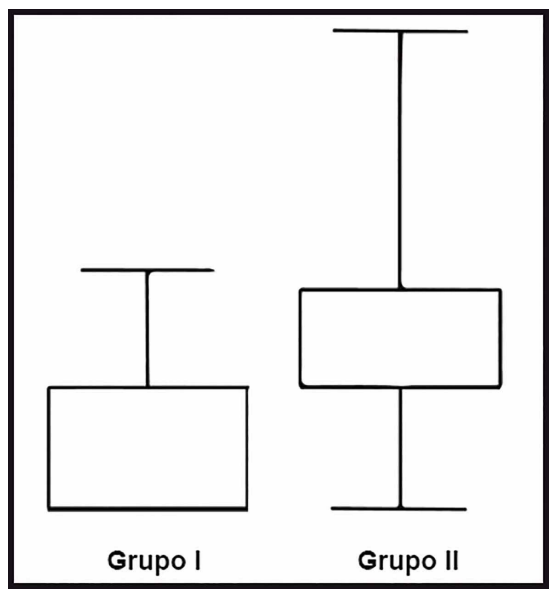

Fig. 2. Comparación en el Grupo I (izquierda) y Grupo II (derecha) según índice gingival (promedio cavidad oral).

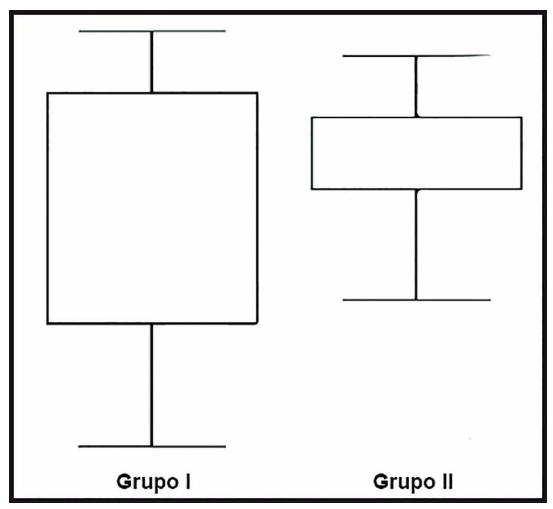

Fig. 3. Comparación en el Grupo I (izquierda) y Grupo II (derecha) según el índice de carga inflamatoria periodontal (PIBI). 
Tabla I. Rango de edad, índice de placa bacteriana, índice gingival e índice carga inflamatoria periodontal de los pacientes incluidos en el estudio.

\begin{tabular}{|c|c|c|c|c|c|c|}
\hline & \multicolumn{2}{|c|}{ Grupo I } & \multicolumn{2}{|c|}{ Grupo II } & \multirow[b]{2}{*}{ Estadístico } & \multirow[b]{2}{*}{ Sig. } \\
\hline & Promedio & DE & Promedio & DE & & \\
\hline Edad & 56,7 & 3,5 & 57,1 & 2,6 & 0,500 & $P>0,05$ \\
\hline IP & 84,8 & 3,5 & 87,0 & 9,8 & 0,440 & $P>0,05$ \\
\hline IG & 1,75 & 0,3 & 1,9 & 0,2 & 0,001 & $p<0,05$ \\
\hline PIBI & 85,00 & 10,0 & 90,9 & 4,8 & 0,001 & $P<0,05$ \\
\hline
\end{tabular}

$\mathrm{IP}=$ Índice de Placa bacteriana (promedio cavidad oral); IG= Índice gingival (promedio cavidad oral); $\mathrm{PIBI}=$ Índice de Carga inflamatoria (promedio cavidad oral).

\section{DISCUSIÓN}

De acuerdo a Fajardo et al. (2010) las estatinas podrían tener un efecto benéfico sobre la pérdida ósea y la movilidad dentaria en pacientes con periodontitis crónica. Lindy et al. encontraron que los pacientes en tratamiento con estatinas y periodontitis crónica presentan menor número de sacos profundos que los sin ella. Pradeep et al. (2012) en un estudio clínico aleatorizado señaló que la aplicación subgingival de simvastatina, se relaciona con menor valor de índice gingival, menor profundidad de sondaje y mayor ganancia de inserción clínica. Se ha evidenciado de acuerdo a estudios realizados en pacientes diabéticos (Sakoda et al., 2006) que las altas concentraciones de glucosa a nivel del fluído gingival crevicular, al igual que los lipopolisacáridos liberados por bacterias gram negativas son capaces de producir la liberación de importantes cantidades de mediadores inflamatorios desde las células del hospedero, cuya liberación puede ser disminuída en presencia de las estatinas. Schmidt et al. (2008) mencionaron que las estatinas interfieren la adhesión de los leucocitos en el fenómeno inflamatorio alterando importantes eventos como la presentación de antígenos, lo que entre otras vías explicaría menores niveles de inflamación en los tejidos. Los estudios elaborados por Kwak et al. (2002), demostraron inhibición in vitro en la expresión de ciertas citoquinas inflamatorias como factor de necrosis tumoral alfa, lo que también explica una menor expresión inflamatoria. Las estatinas podrían disminuir la liberación de metaloproteinasas de la matriz, entre ellas la 1-2-3-9 en células musculares lisas y macrófagos (Luan et al., 2003), se le ha atribuido además la característica de estimular la función osteoblástica, logrando a través de su aplicación tópica la reparación de defectos óseos (Seto et al., 2008). Horiuchi \& Maeda et al. (2006), han señalado que aumentan la expresión de proteína morfogenética ósea, promoviendo la mineralización en osteoblastos en cultivos, indicando un efecto anabólico en el hueso. Yazawa et al. (2005), señalaron que las estatinas pueden inducir la proliferación y diferenciación de células osteoblásticas a partir de células del ligamento periodontal. Kim et al. (2003) mencionaron que la lovastatina y la simvastatina podrían ser útiles en procedimientos de regeneración de tejidos óseos. Existe en consecuencia una gran cantidad de estudios que indican que las estatinas podrían tener un efecto benéfico sobre la enfermedad periodontal lo que es coincidente con los resultados obtenidos en el presente estudio en el cuál se observó una menor inflamación de los tejidos periodontales en los pacientes que se encuentran en tratamiento con atorvastatina sistémica por más de un año.

Según Saver et al. (2007), el uso de las estatinas no estaría en nada relacionado ni con la pérdida dentaria ni con el aumento en el riesgo de sufrir tal evento.

Se necesita mayor cantidad de estudios clínicos aleatorizados que permitan determinar el real efecto de las estatinas en el curso de la enfermedad periodontal.

BRAVO-BOWN, J. \& ULLOA-CARMONA, M. Association of atorvastatin in systemic inflammatory characteristics of chronic periodontitis. Int. J. Odontostomat., 9(3):493-496, 2015.

ABSTRACT: To determine the effect of systemic atorvastatin on gingival index and periodontal inflammatory burden index $(\mathrm{PIBI})$ in patients with chronic periodontitis in an adult population of the northern sector of the city of Antofagasta. This study was conducted in patients referred from the clinic of periodontology of Centro Asistencial Norte under the Department of Health Service of Antofagasta. A group of 60 patients over 40 years old was selected, diagnosed with chronic periodontitis, with at least 14 teeth in the mouth without antibiotic treatment in the last 6 months. 
These patients were divided into two groups; 30 of them were treated with atorvastatin for at least 1 year (Group I) and 30 who were not treated with this drug (Group II). The variables analyzed were simplified plaque index, gingival index and PIBI. The atorvastatin systemic is associated with a decrease in gingival index and $\mathrm{PIBI}$ in adult patients with chronic periodontitis.

KEY WORDS: hydroxymethylglutaryl-coa reductase inhibitors, periodontal index, periodontal diseases.

\section{REFERENCIAS BIBLIOGRÁFICAS}

Armitage, G. C. Development of a classification system for periodontal diseases and conditions. Ann. Periodontol., 4(1):1-6, 1999.

Cunha-Cruz, J.; Saver, B.; Maupome, G. \& Hujoel, P. P. Statin use and tooth loss in chronic periodontitis patients. J. Periodontol., 77(6):1061-6, 2006.

Fajardo, M. E.; Rocha, M. L.; Sánchez-Marin, F. J. \& Espinosa-Chávez, E. J. Effect of atorvastatin on chronic periodontitis: a randomized pilot study. J. Clin. Periodontol., 37(11):1016-22, 2010.

Horiuchi, N. \& Maeda, T. Statins and bone metabolism. Oral Dis., 12(2):85-101, 2006.

Ikeda, U. \& Shimada, K. Statins and monocytes. Lancet, 353(9169):2070, 1999.

Kim, H. J.; Camilleri, M.; McKinzie, S.; Lempke, M. B.; Burton, D. D.; Thomforde, G. M. \& Zinsmeister, A. R. A randomized controlled trial of a probiotic, VSL\#3, on gut transit and symptoms in diarrhoea-predominant irritable bowel syndrome. Aliment. Pharmacol. Ther., 17(7):895-904, 2003.

Kinane, D. F. Causation and pathogenesis of periodontal disease. Periodontol. 2000, 25:8-20, 2001.

Kwak, B.; Mulhaupt, F.; Myit, S. \& Mach, F. Statins as a newly recognized type of immunomodulator. Nat. Med., 6(12):1399-402, 2002

Lindy, O.; Suomalainen, K.; Mäkelä, M. \& Lindy, S. Statin use is associated with fewer periodontal lesions: A retrospective study. BMC Oral Health, 8:16, 2008.

Luan, Z.; Chase, A. J. \& Newby, A. C. Statins inhibit secretion of metalloproteinases-1, $-2,-3$, and -9 from vascular smooth muscle cells and macrophages. Arterioscler. Thromb. Vasc. Biol., 23(5):769-75, 2003.
Nassar, P. O.; Nassar, C. A.; Guimarães, M. R.; Aquino, S. G.; Andia, D. C.; Muscara, M. N.; Spolidorio, D. M.; Rossa, C. Jr. \& Spolidorio, L. C. Simvastatin therapy in cyclosporine A-induced alveolar bone loss in rats. J. Periodontal Res., 44(4):479-88, 2009.

Pradeep, A. R.; Raghavendra, N. M.; Sharma, A.; Patel, S. P.; Raju, A.; Kathariya, R.; Rao, N. S. \& Naik, S. B. Association of serum and crevicular visfatin levels in periodontal health and disease with type 2 diabetes mellitus. J. Periodontol., 83(5):629-34, 2012.

Sakoda, K.; Yamamoto, M.; Negishi, Y.; Liao, J. K.; Node, K. \& Izumi, Y. Simvastatin decreases IL-6 and IL-8 production in epithelial cells. J. Dent. Res., 85(6):5203, 2006.

Saver, B. G.; Hujoel, P. P.; Cunha-Cruz, J. \& Maupomé, G. Are statins associated with decreased tooth loss in chronic periodontitis? J. Clin. Periodontol., 34(3):2149, 2007.

Schmidt, W. M.; Spiel, A. O.; Jilma, B.; Wolzt, M. \& Muller, M. In-vivo effects of simvastatin and rosuvastatin on global gene expression in peripheral blood leucocytes in a human inflammation model. Pharmacogenet. Genomics, 18(2):109-20, 2008.

Seto, H.; Ohba, H.; Tokunaga, K.; Hama, H.; Horibe, M. \& Nagata, T. Topical administration of simvastatin recovers alveolar bone loss in rats. J. Periodontal Res., 43(3):261-7, 2008.

Vaziri, H.; Naserhojjati-Roodsari, R.; Tahsili-Fahadan, N.; Khojasteh, A.; Mashhadi-Abbas, F.; Eslami, B. \& Dehpour, A. R. Effect of simvastatin administration on periodontitis-associated bone loss in ovariectomized rats. J. Periodontol., 78(8):1561-7, 2007.

Yazawa, H.; Zimmermann, B.; Asami, Y. \& Bernimoulin, J. P. Simvastatin promotes cell metabolism, proliferation, and osteoblastic differentiation in human periodontal ligament cells. J. Periodontol., 76(2):295-302, 2005.

Dirección para Correspondencia:

Dr. Joel Bravo Bown

Especialista en Periodoncia

Profesor Asociado

Universidad de Antofagasta

Antofagasta

CHILE

Email: joel.bravo@uantof.cl jbravo@odontologia.uchile.cl

Recibido: 08-09-2015

Aceptado: 28-10-2015 